\title{
Knockdown expression of a MYB-related transcription factor gene, OSMYBS2, enhances production of recombinant proteins in rice suspension cells
}

\author{
Desyanti Saulina Sinaga ${ }^{1,3}$, Shin-Lon Ho ${ }^{2}$, Chung-An Lu³ ${ }^{3}$ Su-May Yu ${ }^{4}$ and Li-Fen Huang ${ }^{1 *}$ (D)
}

\begin{abstract}
Background: Transgenic plant suspension cells show economic potential for the production of valuable bioproducts. The sugar starvation-inducible rice aAmy3 promoter, together with its signal peptide, is widely applied to produce recombinant proteins in rice suspension cells. The OsMYBS2 transcription factor was shown recently to reduce activation of the aAmy3 promoter by competing for the binding site of the TA box of the aAmy3 promoter with the potent OsMYBS1 activator. In this study, rice suspension cells were genetically engineered to silence OsMYBS2 to enhance the production of recombinant proteins.

Results: The mouse granulocyte-macrophage colony-stimulating factor (mGM-CSF) gene was controlled by the aAmy3 promoter and expressed in OsMYBS2-silenced transgenic rice suspension cells. Transcript levels of the endogenous aAmy3 and the transgene MGM-CSF were increased in the OsMYBS2-silenced suspension cells. The highest yield of recombinant mGM-CSF protein attained in the OsMYBS2-silenced transgenic suspension cells was $69.8 \mu \mathrm{g} /$ $\mathrm{mL}$, which is 2.5 -fold that of non-silenced control cells. The yield of recombinant $\mathrm{mGM}$-CSF was further increased to $118.8 \mathrm{\mu g} / \mathrm{mL}$ in cultured cells derived from homozygous $F_{5}$ seeds, which was 5.1 times higher than that of the control suspension cell line.
\end{abstract}

Conclusions: Our results demonstrate that knockdown of the transcription factor gene OsMYBS2 increased the activity of the aAmy3 promoter and improved the yield of recombinant proteins secreted in rice cell suspension cultures.

Keywords: OsMYBS2, Rice suspension cells, Sugar, aAmy3 promoter, Recombinant protein, Mouse GM-CSF

\section{Background}

Plant molecular farming is a technology used in genetic engineering whereby plants are used to produce valuable therapeutic recombinant proteins and secondary metabolites by transferring recombinant gene(s) to plant hosts [1]. With clear advantages in terms of biosafety and the cost of large-scale production, plant molecular farming

*Correspondence: hlf326@saturn.yzu.edu.tw

${ }^{1}$ Graduate School of Biotechnology and Bioengineering, Yuan Ze University, Taoyuan City 320, Taiwan, ROC

Full list of author information is available at the end of the article has received attention as a powerful means of expressing recombinant proteins to yield pharmaceutical products, such as antibodies, enzymes, vaccines, and cytokines [2-4]. However, the application of transgenic plants in the field has raised concerns associated with subsequent purification, contamination of transgenes in the food chain via cross pollination, and strict government regulation of genetically modified crops. Transgenic plant suspension cells are cultured in a controlled sterile environment and can be upscaled using bioreactors, thus showing economic potential for the production of valuable bioproducts $[5,6]$. original author(s) and the source, provide a link to the Creative Commons licence, and indicate if changes were made. The images or other third party material in this article are included in the article's Creative Commons licence, unless indicated otherwise in a credit line to the material. If material is not included in the article's Creative Commons licence and your intended use is not permitted by statutory regulation or exceeds the permitted use, you will need to obtain permission directly from the copyright holder. To view a copy of this licence, visit http://creativecommons.org/licenses/by/4.0/. The Creative Commons Public Domain Dedication waiver (http://creativeco mmons.org/publicdomain/zero/1.0/) applies to the data made available in this article, unless otherwise stated in a credit line to the data. 
For the production of recombinant proteins, the host cells of rice and tobacco are those used most frequently in plant suspension cultures. The best-known system of transgenic rice cell suspension culture is based on the rice $A L P H A-A M Y L A S E 3$ gene $(\alpha A m y 3$, also termed $R A m y 3 D$ ) promoter, which is induced strongly by sugar starvation [7]. The signal peptide of $\alpha$ Amy3 allows recombinant proteins to be secreted into the liquid medium, thereby avoiding cell lysis and the complicated steps required for protein purification [8]. Several recombinant proteins have been produced using the $\alpha A m y 3$ promoter and signal peptide in cultured cells of transgenic rice cell suspensions [9-16]. Although the $\alpha A m y 3$ promoter has been used widely for sugar-regulated recombinant protein production [9], rice cells have been genetically engineered to improve the $\alpha A m y 3$ promoter based-recombinant production system. For example, knockdown of endogenous $\alpha A m y 3$ expression increased recombinant human GM-CSF production 1.9-fold in transgenic rice cells [17]; silencing of the expression of the CYSTEINE PROTEASE gene in transgenic rice cells resulted in an increase in the yield of recombinant human GM-CSF [18].

Sugar signals mediate transcriptional regulation of $\alpha A m y 3$ [7]. A duplicate TA box in the $\alpha A m y 3$ promoter is an essential regulatory motif for potent activation of the $\alpha A m y 3$ promoter in sugar-starved rice cells [7, 19, 20]. The TA box can be bound by three sugar-repressible 1R-MYB transcription factors: OsMYBS1, OsMYBS2, and OsMYBS3 $[21,22]$. Regulation of the promoter activity of $\alpha A m y 3$ is achieved by competitive binding between OsMYBS1 and OsMYBS2 to the TA box of the $\alpha A m y 3$ promoter [22]. OsMYBS1 activates the TA box-containing promoter [21, 23], whereas OsMYBS2 reduces promoter activity in rice cells under sugar depletion [21, 22]. Based on the study of overexpression and underexpression of OsMYBS2 in transgenic rice cells, a lower level of OsMYBS2 is essential for potent activation of the $\alpha A m y 3$ promoter under sugar depletion [22].

Granulocyte-macrophage colony-stimulating factor (GM-CSF) is an immune-response cytokine generated by macrophages, endothelial cells, and immune-stimulated fibroblasts [24-28]. The GM-CSF protein functions in the development and activation of myeloid precursor cells, macrophages, granulocytes, and dendritic cells [29-32]. GM-CSF has been used in various clinical applications, including as a vaccine adjuvant, in cancer therapy, and immunotherapy for malignancies [33-37]. GM-CSF shows species specificity; although it shares $54 \%$ amino acid sequence identity with human GM-CSF, mouse GMCSF (mGM-CSF) is used preferentially in immune system- and cancer-related research.
In a previous study, we produced mGM-CSF using $\alpha A m y 3$ promoter-based transgenic rice suspension cells with mGM-CSF demonstrated to accumulate to a maximum yield of $24.6 \mathrm{mg} / \mathrm{L}$ attained in a 2 L bioreactor [38]. The transcription factor OsMYBS2 reduces activation of the $\alpha A m y 3$ promoter by competing with OsMYBS1 for binding to the TA box of the $\alpha A m y 3$ promoter [22]. Therefore, the culture of genetically engineered rice suspension cells where OsMYBS2 activity is repressed is one potential strategy to increase production of a recombinant protein based on a cell suspension culture system. To evaluate the effectiveness of this strategy, we compared the yield of the recombinant protein mGM-CSF between wild-type (WT) and OsMYBS2-knockdown rice cell suspensions. The yield of recombinant mGMCSF production increased to $118.8 \mu \mathrm{g} / \mathrm{mL}$ in OsMYBS2knockdown cells compared with that seen in WT rice suspension cells. The present results demonstrate that production of the recombinant protein mGM-CSF can be enhanced using OsMYBS2-knockdown transgenic rice cell suspensions.

\section{Materials and methods \\ Plant materials}

Transgenic lines harboring the $\alpha A m y 3 p:: m G M-C S F$ [38] and Ubip::OsMYBS2RNAi [22] transgenes were used in this study. The transgenic lines were in the 'Tainung 67' (TNG67) background and were generated by Agrobacterium-mediated transformation [22, 38]. The $\alpha A m y 3 p:: m G M-C S F$ transgene contains a rice sugar depletion-inducible promoter, $\alpha A m y 3 \mathrm{p}$, and an $\alpha$ Amy3 signal peptide DNA fused upstream of the $m G M-C S F$ gene (Fig. 1A). Recombinant mGM-CSF proteins were sucessfully produced in the $\alpha A m y 3$ promoter-based transgenic rice suspension cells [38]. The Ubip::OsMYBS2RNAi transgene contains an OsMYBS2 RNAi DNA fragment, which is an inverted repeat of the 271-base pair (bp) region at the $3^{\prime}$ untranslated region of $O s M Y B S 2$ cDNA fused at the up- and downstream ends of a GFP coding sequence, under the control of the maize ubiquitin gene (Ubi) promoter (Fig. 1A). Knockdown of OsMYBS2 expression in the Ubip::OsMYBS2RNAi transgenic lines has been reported previously [22].

To obtain single-copy transgene lines, these transgenic lines were selected based on 3:1 ratio of transgene from $\mathrm{T}_{1}$ generation for possible transformation events that the transgene was inserted at single locus in the genome. Genotype and expression levels of transgene were monitored from $\mathrm{T}_{2}$ to $\mathrm{T}_{5}$ generation of the transgenic lines. The $\alpha A m y 3 p:: m G M-C S F$ and Ubip::OsMYBS2RNAi stable homozygous transgenic lines were obtained, respectively. 
(A)

$\underline{\alpha A m y 3:: m G M-C S F \text { construct }}$

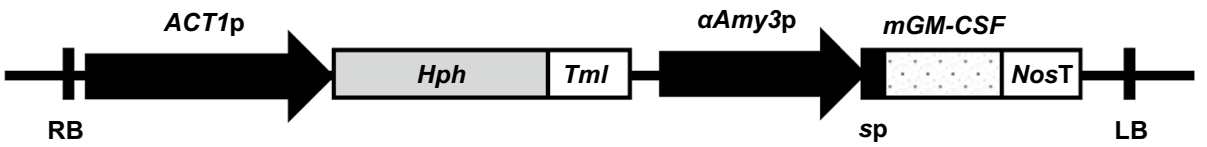

$\underline{U b i: \text { OSMMYBS2RNAi construct }}$

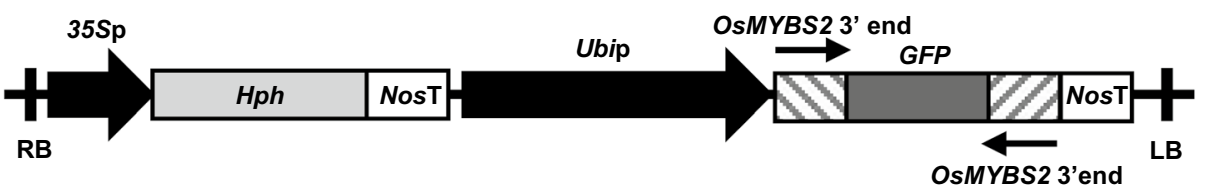

(B)

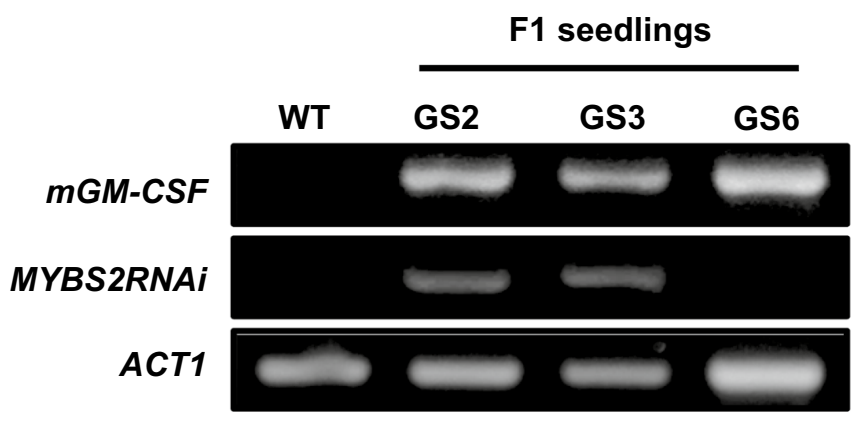

(C)

GS2- ( F2 seedlings)

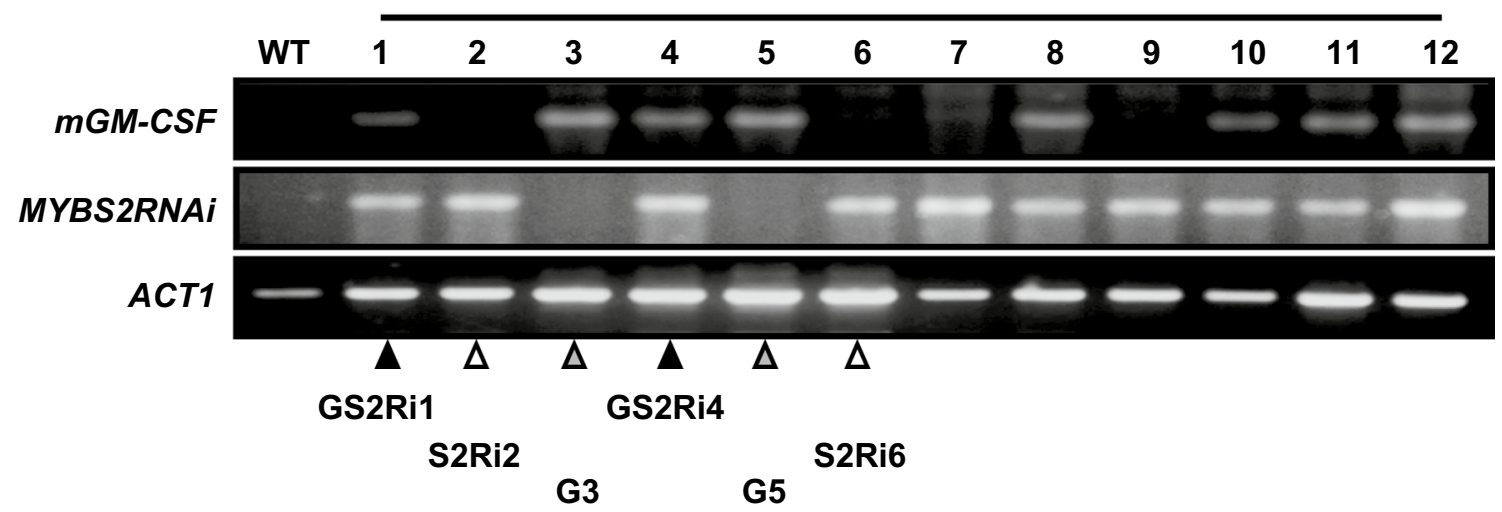

Fig. 1 Generation of transgenic rice plants harboring the aAmy3p::mGM-CSF and Ubip::OSMYBS2RNAi chimeric genes. A Schematic representation of the expression cassettes in transgenic rice plants used for a dihybrid cross. For aAmy3p::mGM-CSF, the mGM-CSF cDNA was inserted downstream of the aAmy3 promoter (aAmy3p)-signal peptide (sp) sequence. For Ubip::OsMYBS2RNAi, a 271 bp fragment at the $3^{\prime}$ end of the OsMYBS2 cDNA was ligated in sense and antisense orientations to the GFP CDNA and fused downstream of the Ubi promoter (Ubip). B and C PCR-based genotype detection of the aAmy3p:: mGM-CSF and Ubip::OSMYBS2RNAi chimeric genes in $\mathrm{F}_{1}$ progeny $(\mathbf{B})$ and $\mathrm{F}_{2}$ population derived from self-pollinated $\mathrm{F}_{1}$ plants (C). The primer sets for $m G M$-CSF and OsMYBS2RNAi were used to amplify specifically the aAmy3p::mGM-CSF and Ubip::OsMYBS2RNAi chimeric genes, respectively. Arrowheads indicate the lines GS2Ri1 and GS2Ri4 (OsMYBS2 knockdown and mGM-CSF expression), S2Ri2 and S2Ri6 (OsMYBS2 knockdown only), and G3 and G10 (mGM-CSF expression only), which were selected for further studies 


\section{Crossing}

The $\alpha A m y 3 p:: m G M-C S F$ homozygous transgenic line was used as the female parent and the Ubip::OsMYBS2RNAi homozygous transgenic line was used as the male parent. A panicle with spikelets from one of the $\alpha A m y 3 p:: m G M$ CSF homozygous transgenic lines was selected to cross. The top one third of each spikelet was cut off using fine pointed sharp scissors. Then the whole panicle was soaked in warm water at $43{ }^{\circ} \mathrm{C}$ for $5 \mathrm{~min}$ to inactivate the pollen harboured by the ather pollen sacs. Stamens were removed gently with fine-tip forceps. Pollination was performed with the pollen that had been sampled from the Ubip::OsMYBS2RNAi homozygous transgenic line to get the F1 hybrid seeds. Progenies of the $\mathrm{F}_{2}, \mathrm{~F}_{3}, \mathrm{~F}_{4}$, and $\mathrm{F}_{5}$ generations were raised by self-pollination.

\section{Establishment of rice cell suspension cultures}

Seeds of the $F_{3}$ and $F_{5}$ progenies were dehulled, sterilized with $3 \%$ Sodium hypochlorite for $30 \mathrm{~min}$, washed extensively with sterile water, placed on Murashige and Skoog (MS) [39] solid medium supplemented with $10 \mu \mathrm{M}$ 2 4-dinitrophenylhydrazine (2,4-D) and 3\% sucrose, and incubated at $28^{\circ} \mathrm{C}$ in the dark. After 1 month, calli were transferred to $25 \mathrm{~mL}$ N6 [40] liquid medium containing $10 \mu \mathrm{M} 2,4-\mathrm{D}$ and $3 \%$ sucrose in a $250 \mathrm{~mL}$ flask and maintained on an orbital shaker at $110 \mathrm{rpm}$ at $28^{\circ} \mathrm{C}$ in a dark culture room. Suspension cells were subcultured every 7 days in fresh N6 liquid medium containing sucrose and 2,4-D.

\section{PCR-base genotype analysis}

Genomic DNA was isolated from 2-week-old seedlings or calli [41]. The rice samples were ground by mortar and pestle with liquid nitrogen. The ground sample powder was transferred into a pre-chilled Eppendorf tube. Then, $0.75 \mathrm{~mL}$ extraction buffer $(100 \mathrm{mM}$ Tris- $\mathrm{HCl}$ pH 8.0, $50 \mathrm{mM}$ EDTA pH 8.0, $100 \mathrm{mM} \mathrm{NaCl}, 1 \%$ SDS, $1 \% \beta$-mercaptoethanol) was added to dissolve the sample powder, and all samples were incubated at $65^{\circ} \mathrm{Cfor}$ $15 \mathrm{~min}$. The sample was mixed with $0.25 \mathrm{~mL} 3 \mathrm{M}$ potassium acetate and incubated at $-20^{\circ} \mathrm{C}$ for $20 \mathrm{~min}$. Then the sample solution was centrifuged at $10,000 \times g$ for $20 \mathrm{~min}$, and post centrifuging, the sample supernatant was collected. One-fifth the volume of isopropanol was added to the supernatant for DNA precipitation. The sample was then centrifuged at $10,000 \times g$ for $20 \mathrm{~min}$ to pellet the DNA. After air-drying, the DNA pellet was dissolved in

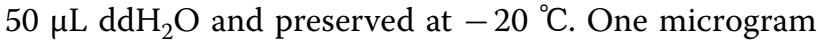
of each genomic DNA sample was subjected to PCR using the following gene-specific primers: for amplification of $m G M-C S F$ DNA, 5'-ATGGCACCCACCCGC TCACC- $3^{\prime}$ and $5^{\prime}$-AAAAAACCAGTCCAAAAATGA- ${ }^{\prime}$; for amplification of OsMYBS2RNAi DNA, 5'-AAAGGA TCCCTCGAGATGGTGAGCAAGGGCGAG- $3^{\prime}$ and 5'-GGGATCCTATCTAGA CTTGTACAGCTCGTC CAT-3'; and for amplification of ACTIN-1 (ACT1) DNA, $5^{\prime}$-CTGATGGACAGGTTATCACC- $3^{\prime}$ and $5^{\prime}$-CAGGTA GCAATAGGTATTACAG- $3^{\prime}$. The PCR products were separated by electrophoresis in $0.5 \mathrm{X}$ TAE buffere at $100 \mathrm{~V}$.

\section{Quantitative real-time polymerase chain reaction (qRT-PCR) analysis}

Total RNA was isolated from caill as described previously [38]. The rice cell sample was ground by mortar and pestle with liquid nitrogen. The ground sample powder was transferred into a pre-chilled Eppendorf tube, and then $0.5 \mathrm{~mL}$ TRIzol Reagent (Invitrogen, Carlsbad, CA, USA) was added to the sample powder and incubated for $10 \mathrm{~min}$ at room temperature. The rice cell sample was mixed with $100 \mu \mathrm{L}$ chloroform and incubated for $10 \mathrm{~min}$ at room temperature. The sample was centrifuged at $10,000 \times g$ for $20 \mathrm{~min}$ at $4{ }^{\circ} \mathrm{C}$ to collect the supernatant. Next, $250 \mu \mathrm{L}$ isopropanol was added to the supernatant to precipitate the total RNA. After $10 \mathrm{~min}$ of incubation at room temperature, the sample was centrifuged at $10,000 \times g$ for $20 \mathrm{~min}$ at $4{ }^{\circ} \mathrm{C}$ to pellet the total RNA. The RNA pellet was washed with $500 \mu \mathrm{L}$ of $75 \%$ ethanol. After removing the $75 \%$ ethanol wash, the RNA pellet was dried at room temperature for $10 \mathrm{~min}$. The RNA pellet was than dissolved in $50 \mu \mathrm{L}$ DEPC-treated water. Isolated total RNA was treated with RNase-free DNase I (NEB, Ipswich, MA, USA) to remove possible DNA contamination. First-strand complementary DNA (cDNA) was synthesized from $2.5 \mu$ g total RNA using ReverTra Ace ${ }^{\circledR}$ reverse transcriptase (Toyobo, Osaka, Japan) with oligo-dT primers. A tenfold dilution of the resultant firststrand cDNA was subjected to qRT-PCR with $m G M$ CSF, OsMYBS2, and $\alpha A m y 3$ gene-specific primers using the FastStart Essential DNA Green Master (Roche, Basel, Switzerland) and the PikoReal ${ }^{\mathrm{TM}}$ Real-Time PCR system (Thermo, Waltham, MA, USA). For detection of $m G M$ CSF mRNA, the aforementioned specific primers for amplification of mGM-CSF DNA were used. The primers $5^{\prime}$-GGACTCGAGAATGGAGCAGCATGAGGA- $3^{\prime}$ and $5^{\prime}$-GTCCATGGTACCCCTTTCTT- $3^{\prime}$ were used for detection of OsMYBS2 mRNA, and the primers 5'-GTA GGCAGGCTCTCTAGCCTCTAGG-3' and 5'-AAC CTGACATTATATATTGCACC-3' were used to detect $\alpha A m y 3$ mRNA. The qPCR procedure was repeated independently at least three times. Expression of $A C T I N-1$ in rice suspension cells is repressed in sugar free medium, so $18 \mathrm{~S}$ rRNA was applied as a reference gene for normalization in detection of gene expression under sugar starvation treatments. The primers used to detect $18 \mathrm{~S}$ rRNA 
were 5'-CCTATCAACTTTCGATGGTAGGATA-3' ${ }^{\prime}$ and 5'-CGTTAAGGGATTTAGATTGTACTCATT- ${ }^{\prime}$. The relative gene expression level was expressed as the ratio of the target gene mRNA abundance to the $18 \mathrm{~S}$ rRNA abundance. Data were analyzed using PikoReal 2.0 software (Thermo).

\section{Western blot analysis}

Protein gel blot analysis was performed as described previously [38] and the concentration of the total protein of each sample was determined by using the Bio-Rad Protein Assay reagent (Bio-Rad, Hercules, CA, USA). The proteins were separated by $15 \%$ SDS-PAGE, and $20 \mu \mathrm{g}$ of protein was loaded per lane. The separated proteins were then transferred onto a PVDF membrane. The polyclonal rabbit anti-mGM-CSF antibody (Abcam, Cambridge, MA, USA) was used as the primary antibody, and HRPconjugated anti-rabbit IgG was used as the secondary antibody to detect rmGM-CSF protein. The signal was detected by chemiluminescence using ECL prime western blot detection (GE Healthcare, Chicago, IL, USA).

\section{Enzyme-linked immunosorbent assay (ELISA)}

The concentration of rmGM-CSF in the culture medium was determined by sandwich ELISA following the method described by Liu et al. [38]. The goat anti-mGMCSF polyclonal antibodies were coated onto 96-well microtiter plates, and then $50 \mu \mathrm{L}$ of cell cultured medium protein samples were added into individual wells of a microtiter plate for $60 \mathrm{~min}$ incubation at $37{ }^{\circ} \mathrm{C}$. The rabbit anti-mGM-CSF polyclonal antibodies (Abcam) were added to the wells and incubated at $37{ }^{\circ} \mathrm{C}$ for $60 \mathrm{~min}$. Goat peroxidase-conjugated anti-rabbit IgG antibodies applied for the detection of rabbit IgG antibody were added and incubated at $37{ }^{\circ} \mathrm{C}$ for $60 \mathrm{~min}$. A substrate of ABTS solution (Sigma, St Louis, MO, USA) was added to the wells. The optical density at $450 \mathrm{~nm}$ of each well was recorded using an Epoch Multi-Volume Spectrophotometer System (BioTek, Winooski, VT, USA).

\section{Results and discussion}

\section{Generation of OsMYBS2 knockdown and $m G M-C S F$} expressing rice plants by a dihybrid cross

Variability of transgene expression is frequently observed in independent transgenic lines due to several factors, such as differences in chromosomal position and transgene copy number. To eliminate genetic background effects on the yield of recombinant mGM-CSF protein between WT and OsMYBS2-knockdown rice suspension cells, a dihybrid cross approach was used to generate the OsMYBS2-knockdown and $m G M$-CSF-expressing transgenic rice plants. The homozygous transgenic rice harboring the $\alpha A m y 3 p:: m G M-C S F$ transgene (Fig. 1A) was used as the female plant, which was crossed with the Ubip::OsMYBS2RNAi (Fig. 1A) homozygous transgenic plant line which was used as the male plant. Given that both parental transgenic rice lines were generated under the genetic background of the rice cultivar Tainung 67 (TNG67) by Agrobacterium-mediated transformation, the progenies derived from the dihybrid crossing event were assumed to have only difference of the transgene copy numbers. Three $F_{1}$ seeds were obtained and the genotype of the $F_{1}$ seedlings was analyzed using PCRbased genotype detection. Two $\mathrm{F}_{1}$ offspring, GS2 and GS3, were dihybrid heterozygous for the OsMYBS2RNA $i$ and $m G M-C S F$ transgenes (Fig. 1B). Subsequently, the $\mathrm{F}_{2}$ progeny were obtained from self-pollination of the GS2 individual. Genotype analysis revealed that four $\mathrm{F}_{2}$ progeny carried the OsMYBS2RNAi gene, namely plants GS2$2,-6,-7$, and -9 , and two F2 progeny were determined to harbor the mGM-CSF transgene, namely plants GS2-3 and -5 . In addition, PCR-based genotyping revealed that six F2 progeny harbored by the mGM-CSF and OsMYBS2RNAi transgenes, including plants GS2-1, -4, $-8,-10,-11$, and -12 (Fig. 1C). For further investigation, GS2-2 and GS2-6 contained the transgene OsMYBS2R$N A i$ and were renamed as S2Ri2 and S2Ri6. Next, GS2-3 and GS2-5 carried the transgene $m G M-C S F$ and were renamed as G3 and G5. Finally, GS2-1 and GS2-4 had both transgenes and were rename as GS2Ri1 and GS2Ri4 (Fig. 1C).

\section{Establishment of OsMYBS2-knockdown} and $m G M-C S F$-expressing transgenic rice suspension cells To investigate the effect of OsMYBS2 knockdown on $\alpha A m y 3 p:: m G M-C S F$ transgene expression, calli were induced from $\mathrm{F}_{3}$ seeds of the WT, two OsMYBS2RNAi only lines, S2Ri2 and S2Ri6, two $m G M-C S F$ only lines, G3 and G5, and two mGM-CSF/OsMYBS2RNAi lines, GS2Ri1 and GS2Ri4. Given that the parental rice lines were generated in the TNG67 background by Agrobacterium-mediated transformation, the progeny of this dihybrid cross were genetically identical, except for the composition of the sequences of the two introduced transgenes. Chen et al. [22] has reported that OsMYBS2 is a negative regulator of $\alpha A m y 3$ expression. Transgenic rice plants constitutively overexpressing OsMYBS2 exhibited reduced seed germination, delayed seedling growth, and shorted mature plant height, while knockdown of OsMYBS2 expression did not show any obvious phenotypes [22]. In this study, callus induction rates from these knockdown transgenic lines were determined to be similar to those of wild-type rice plants. After genotyping of progeny plants (Fig. 2A), calli of these transgenic lines were used to establish cell suspension cultures which exhibited similar cell morphology to WT (Additional 


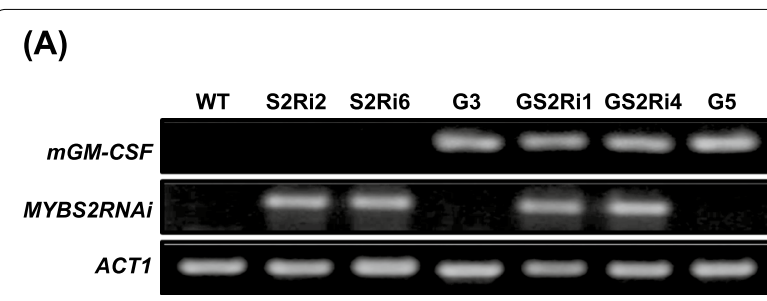

(B)

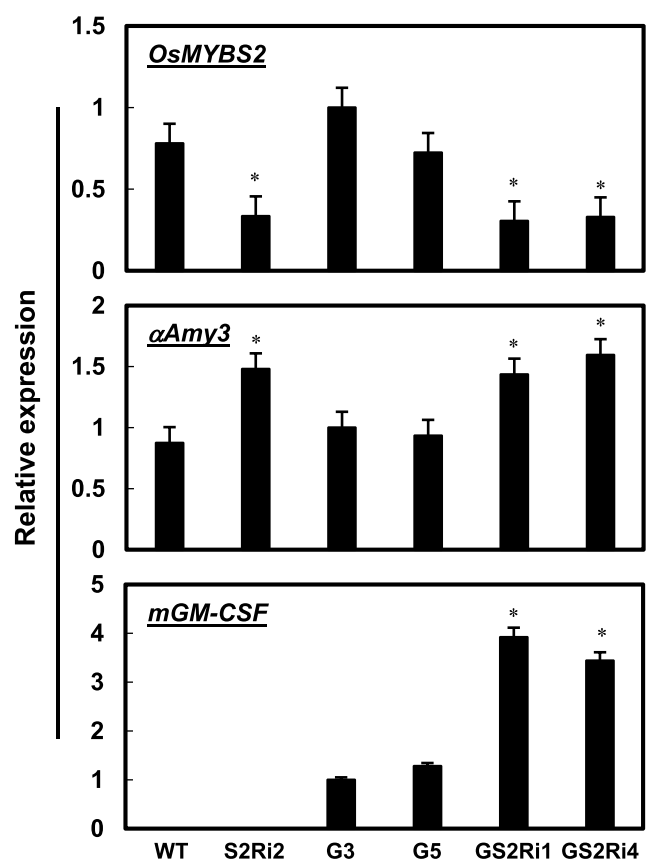

Fig. 2 Establishment and characterization of MGM-CSF/ OsMYBS2RNAi transgenic rice suspension cell lines. A Genotyping of suspension cell lines. PCR-based detection of aAmy3p::mGM-CSF and Ubip::OsMYBS2RNAi chimeric genes was conducted on various calli derived from $\mathrm{F}_{3}$ seeds. The primer sets for $\mathrm{mGM}$-CSF and OSMYBS2RNAi were used to amplify specifically the aAmy3p::mGM-CSF and Ubip::OSMYBS2RNAi chimeric genes, respectively. S2Ri2 and S2Ri6 were OSMYBS2 knockdown lines, G3 and G5 were $m G M$-CSF transgenic lines, and GS2Ri1 and GS2Ri4 were mGM-CSF/OsMYBS2RNAi lines. B Expression of OsMYBS2, aAmy3, and $m G M-C S F$ in the established rice suspension cell lines. Total RNA was isolated from suspension cells sugar-starved for 2 days and then subjected to qRT-PCR using primers specific for OsMYBS2, aAmy3, and mGM-CSF. Error bars indicate the standard deviation (SD) of triplicate experiments. For OSMYBS2 and aAmy3, gene expression was relative to that of wild-type (WT) suspension cells, where $1=$ equivalent. For $m G M-C S F$, gene expression was relative to that of $G 3$ suspension cells, where $1=$ equivalent. ${ }^{*}$ Significantly different from the control cell lines (Student's $t$-test: $p<0.05$ )

file 1: Fig. S1). Subsequently, $m G M-C S F$ mRNA levels were compared in these cell suspension lines under sugar starvation. In addition to the WT control cell line, one of OsMYBS2RNAi only cell lines, S2Ri2, was selected as another control for the knockdown genetic background of OsMYBS2. Total RNA was isolated from the WT, S2Ri2, G3, G5, GS2Ri1, and GS2Ri4 cell suspension lines sugar-starved for $48 \mathrm{~h}$ and subjected to qRTPCR analysis. Expression of OsMYBS2 was lower in the S2Ri2, GS2Ri1, and GS2Ri4 lines than in the WT, G3, and G5 cell lines, as expected (Fig. 2B). The expression of $m G M-C S F$ transgene was controlled by the sugarstarvation-inducible $\alpha A m y 3$ promoter, so mRNA of the transgene was detected only in the sugar-starved G3, G5, GS2Ri1, and GS2Ri4 cell lines (Fig. 2B). Comparison of the various cell lines revealed that $m G M-C S F$ mRNA levels were significantly higher in both $m G M-C S F / O s M Y B$ S2RNAi lines, GS2Ri1 and GS2Ri4, than in the G3 and G5 cell lines (Fig. 2B). Similarly, the $\alpha A m y 3$ mRNA levels were higher in the S2Ri2, GS2Ri1, and GS2Ri4 cell lines than in the WT, G3, or G5 cell lines (Fig. 2B). These results indicate that knockdown of OsMYBS2 increased the expression of the $\alpha A m y 3 p:: m G M-C S F$ transgene in sugar-starved cells.

\section{Knockdown of OSMYBS2 expression increases production} of recombinant $\mathrm{mGM}$-CSF protein in the culture medium To examine whether knockdown of OsMYBS2 expression enhanced production of the recombinant mGM-CSF (rmGM-CSF) protein, the rmGM-CSF protein productivity was compared among two $m G M-C S F$ control cell lines, G3 and G5, and two mGM-CSF/OsMYBS2-RNAi cell lines, GS2Ri1 and GS2Ri4. Cell suspensions were cultured in sugar-containing N6 medium for 3 days, and then $1.0 \mathrm{~mL}$ of cultured cells was incubated in $2.0 \mathrm{~mL}$ sugar-free N6 medium for 5 and 7 days. The cultured medium of each cell line was collected and an equal amount of total medium protein from each line was analyzed by western blot analysis. After sugar starvation for 5 days, the amount of recombinant mGM-CSF (rmGM-CSF) protein detected in the sugar-free liquid N6 medium of the GS2Ri1 and GS2Ri4 cell lines was considerably higher than that detected in the medium of the control cell lines G3 and G5 (Fig. 3). Higher amounts of $\alpha$ Amy 3 protein were detected in the medium of the GS2Ri1 and GS2Ri4 cell lines than in that of the G3 and G5 cell lines (Fig. 3). Similar results for enhanced rmGMCSF production in OsMYBS2-knockdown cell lines were obtained after sugar starvation for 7 days (Additional file 1: Fig. S2). These results indicate that knockdown of OsMYBS2 expression improved rmGM-CSF production in the medium of sugar-starved rice cell suspension cultures.

The OsMYBS2 protein acts as a weak transcription activator that competes to bind to the TA box of the $\alpha A m y 3$ promoter with the strong transcription activator, OsMYBS1, and leads to the low activity of the $\alpha A m y 3$ promoter $[21,22]$. In sugar-starved 


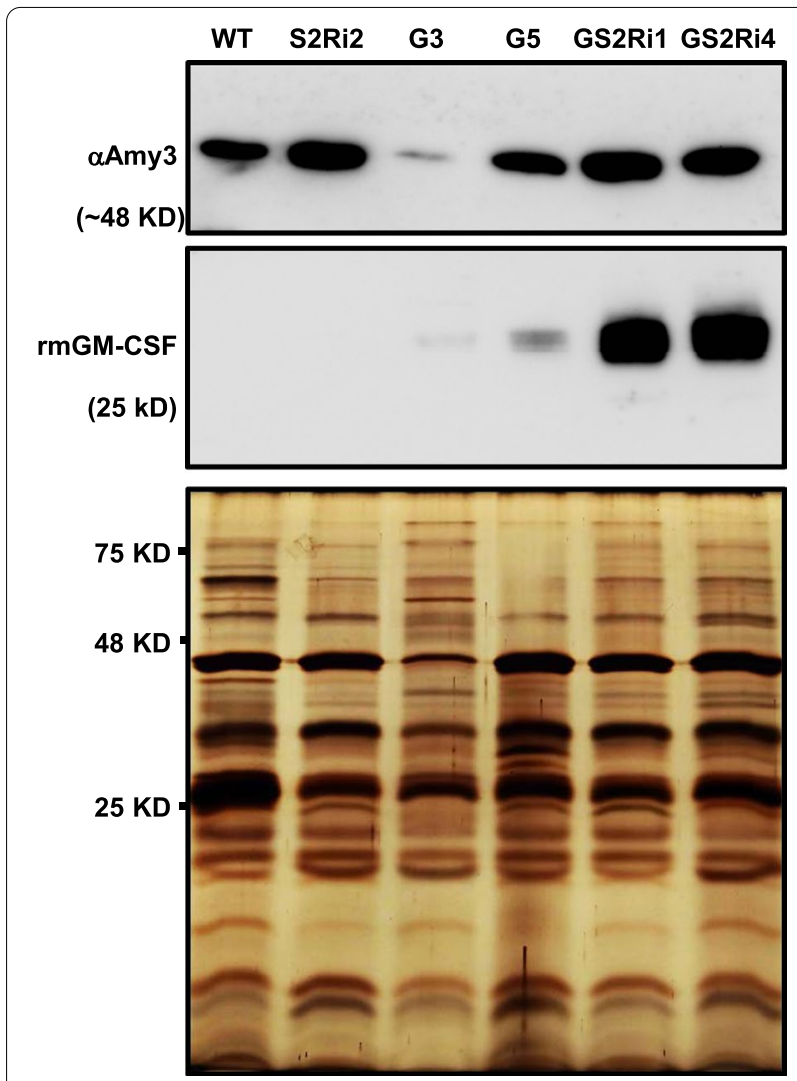

Fig. 3 Production of recombinant $\mathrm{mGM}$-CSF in the culture medium of $m G M-C S F / O s M Y B S 2 R N A i$ transgenic rice suspension cell lines. One milliliter of suspension cells, consisting of the wild type (WT), one OsMYBS2 knockdown line S2Ri2, two MGM-CSF gene transgenic lines G3 and G5, and two OsMYBS2 knockdown and $m G M$-CSF transgenic lines GS2Ri1 and GS2Ri4, were cultured in $2 \mathrm{~mL}$ sugar-free N6 medium for 5 days. Samples of the culture medium were collected to determine $a$ Amy 3 and rmGM-CSF abundance by western blot analysis with specific antibodies to aAmy 3 and mGM-CSF, respectively. Silver staining was used to and visualize bands in the culture medium and represent as the loading control rice suspension cells, OsMYBS2 is relieved from the TA box, while SnRK1A regulates OsMYBS1 binding to the TA box to stimulate activation of the $\alpha A m y 3$ promoter $[22,42]$. The present results show that $m G M$ $C S F$ mRNA levels and recombinant mGM-CSF protein were more abundant in the $m G M-C S F / O s M Y B S 2 R N A i$ suspension cell lines than in the $m G M-C S F$ control cell lines under sugar starvation (Fig. 3). This finding indicates that OsMYBS2 knockdown enhanced $\alpha A m y 3$ promoter activity, and transcription of the $\alpha A m y 3 p:: m G M-C S F$ transgene was increased, thereby imporoving recombinant mGM-CSF production from sugar-starved rice suspension cells.
(A)

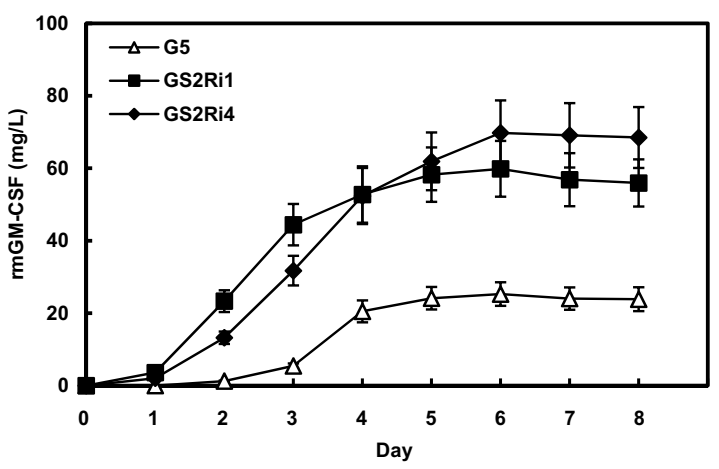

(B)
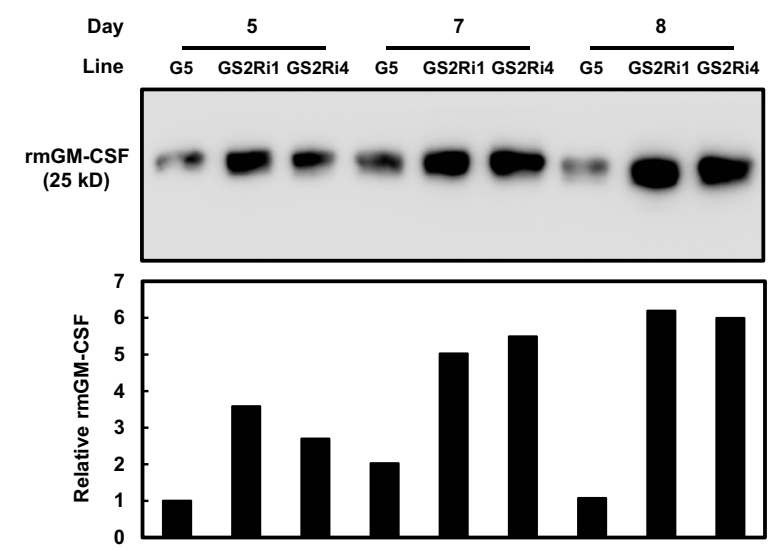

Fig. 4 Recombinant mGM-CSF production profiling in the culture medium of MGM-CSF/OSMYBS2RNAi transgenic rice suspension cell lines. A One milliliter of suspension cells were cultured in $2 \mathrm{~mL}$ sugar-free $\mathrm{N} 6$ medium for various periods. Samples of the culture medium were collected to determine the concentration of rmGM-CSF by ELISA. Error bars represent the SD from triplicate cultures. B Sample of the culture medium collected at days 5, 7, and 8 , and an equal amount of total medium protein from each sample were subjected to western blot analysis using mGM-CSF antibodies. The relative levels of $r m G M-C S F$ abundance were measured using ImageJ software. The rmGM-CSF level was relative to that of G5 suspension cells at day 5 , where $1=$ equivalent

Profiling of rmGM-CSF production in the culture medium of aAmy3p::mGM-CSF/OsMYBS2RNAi rice suspension cells To determine the optimal period for rmGM-CSF production, rice suspension cells at an initial density of $50 \%(\mathrm{v} / \mathrm{v})$ were cultured in sugar-free N6 medium for various periods and protein yields of rmGM-CSF were monitored using an enzyme-linked immunoassay (ELISA). Similar to a previous report [38], rmGM-CSF was detected initially from day 2 , and the yield increased to a maximum of $25.28 \mathrm{mg} / \mathrm{L}$ on day 5 and was maintained until day 8 in the G5 control line (Fig. 4A). The rmGM-CSF protein was detected initially from day 1 in the culture medium of the GS2Ri1 and GS2Ri4 cell lines (Fig. 4A). The 
concentration of rmGM-CSF produced by the GS2Ri1 and GS2Ri4 cell lines increased rapidly and attained maximal concentrations of 59.84 and $69.77 \mathrm{mg} / \mathrm{L}$, respectively, on day 6 to day 8 (Fig. 4A). Compared with the G5 control cell line, the GS2Ri1 and GS2Ri4 suspension cells produced 2.4-2.9 times higher amounts of rmGMCSF in the sugar-containing culture medium, regardless of culture duration (4, 5, 6, 7, and 8 days) (Fig. 4A). To confirm this conclusion, the rmGM-CSF abundance was compared between the G5 and two $\alpha A m y 3 p:: m G M$ CSF/OsMYBS2-RNAi lines by western blot analysis with mGM-CSF antibodies. The relative abundance of rmGMCSF was measured using ImageJ software. Regardless of the sugar-free culture medium from days 5,7 and 8 , the rmGM-CSF abundance in the GS2Ri1 and GS2Ri4 cell lines was at least twofold more abundant than it was in the G5 suspension cells (Fig. 4B). These results indicate that knockdown of OsMYBS2 expression led not only to an increase in rmGM-CSF abundance but also to earlier production in the culture medium.

In the rice suspension culture recombinant protein expression system, cells are initially generally cultured in a sugar-containing medium for cell proliferation and growth. The recombinant protein is produced after cells are transferred to a sugar-free medium. However, when recombinant proteins are produced from sugar-starved cells, the cell viability decreases $[12,38,43]$. We observed that knockdown of OsMYBS2 expression accelerated the accumulation of rmGM-CSF protein in the medium, which may be caused by rapid stimulation of $\alpha A m y 3$ promoter activity in response to sugar depletion in the presence of reduced OsMYBS2 abundance. Acceleration of the $\alpha A m y 3$ promoter response to sugar depletion is particularly advantageous for production of recombinant proteins by the $\alpha A m y 3$ promoter-based recombinant protein expression system because it shortens the duration of recombinant protein production and reduces cell damage. In addition, recombinant protein production is maintained at relatively stable levels under repeated cycles of the presence and absence of sugar by the same batch of rice suspension cells [12, 43]. An early and rapid response to sugar depletion also provides the basis to accelerate the rmGM-CSF production cycle using a repeated-cycle culture strategy in bioreactors.

\section{Stable enhancement of rmGM-CSF production from aAmy3p::mGM-CSF/OsMYBS2RNAi rice suspension cells derived from $\mathrm{F}_{3}$ and $\mathrm{F}_{5}$ seeds}

A dihybrid cross was used to produced trangenic rice plants containing both $\alpha A m y 3 p:: m G M-C S F$ and Ubip:: OsMYBS2RNAi transgenes. Based on genetic laws, $\mathrm{F}_{1}$ progeny should be heterozygous at both loci (OsMYB$S 2 R N A i$ and $m G M-C S F$ transgenes), so only one copy of each transgene existed in $F_{1}$ seeds. After self-pollination of $F_{1}$ plants, dihybrid homozygous transgenic rice plants that contain two copies of each transgenes, OsMYB$S 2 R N A i$ and $m G M-C S F$, can be obtained in $\mathrm{F}_{2}$ population. Genotype analysis of progeny from self-pollinated $\mathrm{F}_{2}$ plants revealed that all 86 analyzed individuals from either GS2Ri1 or GS2Ri4 parents were $m G M$-CSF only or mGM-CSF/OsMYBS2RNAi transgenic lines (Additional file 1: Table S1). This result suggests that progeny in the $\mathrm{F}_{3}$ population was homozygous for the $m G M$ CSF transgene, but remained heterozygous for the $O S M Y B S 2 R N A i$ transgene. Genotype analysis of the $\mathrm{F}_{4}$ progeny showed that both $m G M-C S F$ and OsMYBS2RNAi transgenes were homozygous in the GS2Ri1-2 and GS2Ri1-4 populations (Additional file 1: Table S1). Subsequently, progeny homozygous for $m G M-C S F$ and OsMYBS2RNAi transgenes were obtained in the $\mathrm{F}_{5}$ populations, which were derived from self-pollination of GS2Ri1-2-1, GS2Ri4-1-2, and GS2Ri4-4-1 plants (Additional file 1: Table S1 and Fig. S3).

Gene dosage is correlated with expression level and, in the majority of cases, an increase in gene copy number enhances the expression of genes [44]. Suspension cell lines of GS2Ri1 and GS2Ri4 were derived from the $\mathrm{F}_{2}$ seed population, in which the $m G M-C S F$ and OsMYB$S 2 R N A i$ transgenes were either homozygous or heterozygous. Therefore, GS2Ri1 and GS2Ri4 cells must contain at least one copy of the $m G M-C S F$ and OsMYB$S 2 R N A i$ transgenes. To test whether increased dosage of the $m G M-C S F$ and OsMYBS2RNAi transgenes in rice suspension cells can further increase production of rmGM-CSF, homozygous $\mathrm{F}_{5}$ seeds were used to establish suspension cell lines of GS2Ri1-2-1 and GS2Ri4-1-2, both containing two copies of the $m G M-C S F$ and OsMYBS2R$N A i$ transgenes. Western blot analysis and ELISA were used to compare the production of rmGM-CSF with the control cell line G5 and the $\mathrm{F}_{3}$ seed-derived cell line GS2Ri1. The concentration of rmGM-CSF in the culture medium of the GS2Ri1-2-1 and GS2Ri4-1-2 cell lines after sugar depletion for 5 days was 95.6 and $118.8 \mathrm{mg} / \mathrm{L}$, which were 4 and 5.1 times higher than that of the G5 control cell line, respectively (Fig. 5). This result indicates that the yield of rmGM-CSF was further increased using $\mathrm{F}_{5}$ seed-derived suspension cell cultures.

Various strategies have been used to improve recombinant protein productivity in the rice suspension cell culture system based on the $\alpha A m y 3$ promoter and signal peptide. Liu et al. $[38,43]$ used a one-step cultivation strategy in which the medium was not changed, but rather sucrose became depleted naturally, to improve recombinant protein production in rice suspension cell cultures. Knockdown of either $\alpha A m y 3$ or CysP by RNAi increased the yield of human GM-CSF recombinant 


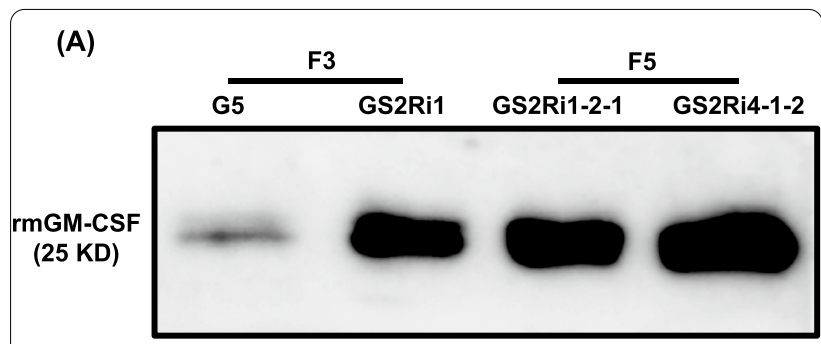

(B)

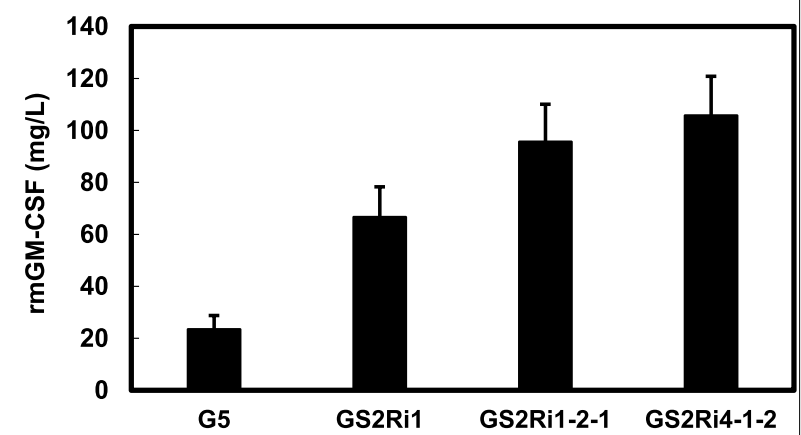

Fig. 5 Production of rmGM-CSF in the $F_{5}$ generation of $m G M-C S F /$ OsMYBS2RNAi transgenic rice suspension cell lines. One milliliter of suspension cells, consisting of G5 ( $m G M$-CSF only) and GS2Ri ( $M G M-C S F / O S M Y B S 2 R N A i)$, both derived from $\mathrm{F}_{3}$ seeds, and GS2Ri1-2-1-1 and GS2Ri4-1-2-2 (both mGM-CSF/OsMYBS2RNAi), derived from $\mathrm{F}_{5}$ seeds, were cultured in $2 \mathrm{~mL}$ sugar-free $\mathrm{N} 6$ medium for 5 days. A Samples of culture medium were collected and equal amounts of total medium protein from each sample were subjected to western blot analysis using mGM-CSF antibodies. B The amount of rmGM-CSF was measured in the culture medium by ELISA. Error bars represent the SD from triplicate cultures

protein by approximately 2.5 -fold. $[17,18]$. The present study shows that rmGM-CSF production was enhanced by a factor of 5.1 using an OsMYBS2 gene-silencing strategy. A strategy that combines all previous approaches is expected to further improve recombinant protein production in rice suspension cells. In addition to the $\alpha A m y 3$ promoter, a modified rice $\alpha A m y 8$ promoter has been used to produce a recombinant human epidermal growth factor in rice suspension cells and seedlings [45]. Knockdown of OsMYBS2 expression increases the mRNA level of $\alpha A m y 8$ in rice suspension cells [22], the promoter of which also contains a TA box [7]. Therefore, an increase in the production of recombinant protein derived from $\alpha A m y 8$ promoter activity can be expected using an OsMYBS2 gene-silencing strategy.

\section{Conclusions}

The function of the rice transcription factor OsMYBS2 underlies the mechanism of sugar regulation of the $\alpha A m y 3$ promoter. Reduction of OsMYBS2 expression is essential for the strong activation of the $\alpha A m y 3$ promoter in rice suspension cells under conditions of sugar depletion. On this basis, through knockdown of OsMYBS2 expression, $\alpha A m y 3$ promoter activity can be increased, thereby facilitating and accelerating the accumulation of secreted recombinant proteins in rice cell suspension cultures.

\section{Abbreviations}

mGM-CSF: Mouse granulocyte-macrophage colony-stimulating factor; rmGM-CSF: Recombinant protein mGM-CSF; aAmy3: Alpha-amylase 3; WT: Wild type; aAmy3p: aAmy3 Promoter; Ubip: Maize ubiquitin promoter; GFP: Green fluorescent protein; 2,4-D: 2 4-Dinitrophenylhydrazine; PCR: Polymerase chain reaction; ACT1: Rice actin 1 gene; qRT-PCR: Quantitative real-time polymerase chain reaction; ELISA: Enzyme-linked immunosorbent assay.

\section{Supplementary Information}

The online version contains supplementary material available at https://doi. org/10.1186/s13007-021-00799-2.

Additional file 1: Table S1. Genotyping result of seedlings. Fig. S1. Suspension rice cell morphology of the WT, two OsMYBS2RNAi only lines, S2Ri2 and S2Ri6, two mGM-CSF only lines, G3 and G5, and two mGMCSF/OsMYBS2RNAi lines, GS2Ri1 and GS2Ri4. Fig. S2. Abundance of rmGMCSF in MGM-CSF/OsMYBS2RNAi transgenic rice suspension cell lines was higher than that in mGM-CSF only transgenic lines. Fig. S3. PCR-based detection of aAmy3::mGM-CSF and Ubi::OSMYBS2RNAi chimeric genes in selected F5 progeny derived from self-pollination of the F4 population.

\section{Acknowledgements}

This work was financially supported (in part) by the Advanced Plant Biotechnology Center at National Chung Hsing University from "The Featured Areas Research Center Program within the framework of the Higher Education Sprout Project" by the Ministry of Education in Taiwan.

\section{Authors' contributions}

LFH and CAL designed the research and wrote the manuscript; DSS and SLH performed the experiments; SLH and SMY manipulated plant materials. All authors read and approve the final manuscript.

\section{Funding}

This work was supported by Grants (103-2313-B-155-001-MY3, 106-2313-B-155001-MY3, and 109-2311-B-008-004-MY3) from the Ministry of Science and Technology of the Republic of China.

\section{Availability of data and materials}

All data generated or analyzed during this study are included in this published article and its supplementary imformation.

\section{Declarations}

Ethics approval and consent to participate

Not applicable.

\section{Consent for publication}

Not applicable.

\section{Competing interests}

The authors have no conflicts of interest to declare.

\section{Author details}

${ }^{1}$ Graduate School of Biotechnology and Bioengineering, Yuan Ze University, Taoyuan City 320, Taiwan, ROC. ${ }^{2}$ Department of Agronomy, National Chiayi University, Chiayi City 600, Taiwan, ROC. ${ }^{3}$ Department of Life Sciences, National 
Central University, Taoyuan City 320, Taiwan, ROC. ${ }^{4}$ Institute of Molecular Biology, Academia Sinica, Nankang, Taipei City 115, Taiwan, ROC.

Received: 23 February 2021 Accepted: 12 September 2021 Published online: 25 September 2021

\section{References}

1. Ma JK, Drake PM, Christou P. The production of recombinant pharmaceutical proteins in plants. Nat Rev Genet. 2003;4:794-805

2. Ahmad P, Ashraf M, Younis M, Hu X, Kumar A, Akram NA, et al. Role of transgenic plants in agriculture and biopharming. Biotechnol Adv. 2012;30:524-40.

3. Daniell H, Singh ND, Mason H, Streatfield SJ. Plant-made vaccine antigens and biopharmaceuticals. Trends Plant Sci. 2009;14:669-79.

4. , Sabalza M, Christou P, Capell T. . Recombinant plant-derived pharmaceutical proteins: current technical and economic bottlenecks. Biotechnol Lett. 2014;36:2367-79.

5. Hellwig S, Drossard J, Twyman RM, Fischer R. Plant cell cultures for the production of recombinant proteins. Nat Biotechnol. 2004;22:1415-22.

6. Sharp JM, Doran PM. Strategies for enhancing monoclonal antibody accumulation in plant cell and organ cultures. Biotechnol Prog. 2001;17:979-92.

7. Lu CA, Lim EK, Yu SM. Sugar response sequence in the promoter of a rice alpha-amylase gene serves as a transcriptional enhancer. J Biol Chem. 1998;273:10120-31.

8. Chen MH, Huang LF, Li HM, Chen YR, Yu SM. Signal peptide-dependent targeting of a rice alpha-amylase and cargo proteins to plastids and extracellular compartments of plant cells. Plant Physiol. 2004;135:1367-77.

9. Kuo YC, Tan CC, Ku JT, Hsu WC, Su SC, Lu CA, et al. Improving pharmaceutical protein production in Oryza sativa. Int J Mol Sci. 2013;14:8719-39.

10. Hong SY, Kwon TH, Jang YS, Kim SH, Yang MS. Production of bioactive human granulocyte-colony stimulating factor in transgenic rice cell suspension cultures. Protein Expr Purif. 2006;47:68-73.

11. Huang J, Wu L, Yalda D, Adkins Y, Kelleher SL, Crane M, et al. Expression of functional recombinant human lysozyme in transgenic rice cell culture. Transgenic Res. 2002;11:229-39.

12. Huang LF, Liu YK, Lu CA, Hsieh SL, Yu SM. Production of human serum albumin by sugar starvation induced promoter and rice cell culture. Transgenic Res. 2005;14:569-81.

13. Jung JW, Kim NS, Jang SH, Shin YJ, Yang MS. Production and characterization of recombinant human acid alpha-glucosidase in transgenic rice cell suspension culture. J Biotechnol. 2016;226:44-53.

14. Lee SJ, Park Cl, Park MY, Jung HS, Ryu WS, Lim SM, et al. Production and characterization of human CTLA4Ig expressed in transgenic rice cell suspension cultures. Protein Expr Purif. 2007;51:293-302.

15. Nam HJ, Kwon JY, Choi HY, Kang SH, Jung HS, Kim DI. Production and purification of recombinant glucocerebrosidase in transgenic rice cell suspension cultures. Appl Biochem Biotechnol. 2017;181:1401-15.

16. Van Giap D, Jung JW, Kim NS. Production of functional recombinant cyclic citrullinated peptide monoclonal antibody in transgenic rice cell suspension culture. Transgenic Res. 2019;28:177-88.

17. Kim NS, Kim TG, Jang YS, Shin YJ, Kwon TH, Yang MS. Amylase gene silencing by RNA interference improves recombinant hGM-CSF production in rice suspension culture. Plant Mol Biol. 2008;68:369-77.

18. Kim NS, Kim TG, Kim OH, Ko EM, Jang YS, Jung ES, et al. Improvement of recombinant hGM-CSF production by suppression of cysteine proteinase gene expression using RNA interference in a transgenic rice culture. Plant Mol Biol. 2008;68:263-75.

19. Chen PW, Lu CA, Yu TS, Tseng TH, Wang CS, Yu SM. Rice alpha-amylase transcriptional enhancers direct multiple mode regulation of promoters in transgenic rice. J Biol Chem. 2002;277:13641-9.

20. Hwang YS, Karrer EE, Thomas BR, Chen L, Rodriguez RL. Three cis-elements required for rice alpha-amylase Amy3D expression during sugar starvation. Plant Mol Biol. 1998;36:331-41.

21. Lu CA, Ho TH, Ho SL, Yu SM. Three novel MYB proteins with one DNA binding repeat mediate sugar and hormone regulation of alpha-amylase gene expression. Plant Cell. 2002;14:1963-80.

22. Chen YS, Ho TD, Liu L, Lee DH, Lee CH, Chen YR, et al. Sugar starvationregulated MYBS2 and 14-3-3 protein interactions enhance plant growth, stress tolerance, and grain weight in rice. Proc Natl Acad Sci USA. 2019;116:21925-35.
23. Hong YF, Ho TH, Wu CF, Ho SL, Yeh RH, Lu CA, et al. Convergent starvation signals and hormone crosstalk in regulating nutrient mobilization upon germination in cereals. Plant Cell. 2012;24:2857-73.

24. Khatami S, Brummer E, Stevens DA. Effects of granulocyte-macrophage colony stimulating factor (GM-CSF) in vivo on cytokine production and proliferation by spleen cells. Clin Exp Immunol. 2001;125:198-201.

25. Rho CR, Park MY, Kang S. Effects of granulocyte-macrophage colony-stimulating (GM-CSF) factor on corneal epithelial cells in corneal wound healing model. PLoS ONE. 2015;10(9):e0138020.

26. Shi FS, Weber S, Gan J, Rakhmilevich AL, Mahvi DM. Granulocyte-macrophage colony-stimulating factor (GM-CSF) secreted by cDNA-transfected tumor cells induces a more potent antitumor response than exogenous GM-CSF. Cancer Gene Ther. 1999;6:81-8.

27. Theoret JK, Jadavji NM, Zhang M, Smith PD. Granulocyte macrophage colony-stimulating factor treatment results in recovery of motor function after white matter damage in mice. Eur J Neurosci. 2016;43:17-24.

28. Wicks IP, Roberts AW. Targeting GM-CSF in inflammatory diseases. Nat Rev Rheumatol. 2016;12:37-48.

29. Shi Y, Liu CH, Roberts Al, Das J, Xu G, Ren G, et al. Granulocyte-macrophage colony-stimulating factor (GM-CSF) and T-cell responses: what we do and don't know. Cell Res. 2006;16:126-33.

30. Vellenga E, Young DC, Wagner K, Wiper D, Ostapovicz D, Griffin JD. The effects of GM-CSF and G-CSF in promoting growth of clonogenic cells in acute myeloblastic leukemia. Blood. 1987;69:1771-6.

31. Hamilton JA, Anderson GP. GM-CSF Biology. Growth Factors. 2004;22:225-31.

32. Armitage JO. Emerging applications of recombinant human granulocytemacrophage colony-stimulating factor. Blood. 1998;92:4491-508.

33. Dale DC, Liles WC, Summer WR, Nelson S. Review: granulocyte colonystimulating factor-role and relationships in infectious diseases. J Infect Dis. 1995;172:1061-75.

34. Wen Q, Xiong WJ, He JC, Zhang SM, Du XL, Liu SD, et al. Fusion cytokine IL-2-GMCSF enhances anticancer immune responses through promoting cell-cell interactions. JTransl Med. 2016;14:1-3.

35. Metcalf D. Control of granulocytes and macrophages: molecular, cellular, and clinical aspects. Science. 1991;254:529-33.

36. Yan WL, Shen KY, Tien CY, Chen YA, Liu SJ. Recent progress in GM-CSF-based cancer immunotherapy. Immunotherapy. 2017;9(4):347-60.

37. Yu TW, Chueh HY, Tsai CC, Lin CT, Qiu JT. Novel GM-CSF-based vaccines: one small step in GM-CSF gene optimization, one giant leap for human vaccines. Hum Vaccin Immunother. 2016;12:3020-8.

38. Liu YK, Huang LF, Ho SL, Liao CY, Liu HY, Lai YH, et al. Production of mouse granulocyte-macrophage colony-stimulating factor by gateway technology and transgenic rice cell culture. Biotechnol Bioeng. 2012;109:1239-47.

39. Murashige T, Skoog F. A revised medium for rapid growth and bioassays with tobacco tissue cultures. Physiol Plant. 1962;15:473-97.

40. Chu CC, Wang CC, Sun CS, Hsu C, Yin KC, Chu CY, Bi FY, et al. Establishment of an efficient medium for anther culture of rice through comparative experimentation on nitrogen sources. SciSn. 1975;18:659-68.

41. Huang LF, Liu YK, Su SC, Lai CC, Wu CR, Chao TJ, et al. Genetic engineering of transitory starch accumulation by knockdown of OsSEX4 in rice plants for enhanced bioethanol production. Biotechnol Bioeng. 2020;1 17:933-44.

42. Lu CA, Lin CC, Lee KW, Chen JL, Huang LF, Ho SL, et al. The SnRK1A protein kinase plays a key role in sugar signaling during germination and seedling growth of rice. Plant Cell. 2007;19:2484-99.

43. Liu YK, Li YT, Lu CF, Huang LF. Enhancement of recombinant human serum albumin in transgenic rice cell culture system by cultivation strategy. $\mathrm{N}$ Biotechnol. 2015;32:328-34.

44. Hood EE, Requesens DV. Recombinant protein production in plants: challenges and solutions. Methods Mol Biol. 2012;824:469-81.

45. Wu CS, Kuo WT, Chang CY, Kuo JY, Tsai YT, Yu SM, et al. The modified rice alphaAmy8 promoter confers high-level foreign gene expression in a novel hypoxia-inducible expression system in transgenic rice seedlings. Plant Mol Biol. 2014;85:147-61.

\section{Publisher's Note}

Springer Nature remains neutral with regard to jurisdictional claims in published maps and institutional affiliations. 